This item was submitted to Loughborough's Research Repository by the author.

Items in Figshare are protected by copyright, with all rights reserved, unless otherwise indicated.

\title{
LES of high speed jet flow from convergent-divergent rectangular S-bend ducts using synthetic inlet conditions
}

PLEASE CITE THE PUBLISHED VERSION

http://dx.doi.org/10.2514/6.2013-2843

\section{PUBLISHER}

AIAA / @ The Authors

VERSION

AM (Accepted Manuscript)

\section{PUBLISHER STATEMENT}

This work is made available according to the conditions of the Creative Commons Attribution-NonCommercialNoDerivatives 4.0 International (CC BY-NC-ND 4.0) licence. Full details of this licence are available at: https://creativecommons.org/licenses/by-nc-nd/4.0/

\section{LICENCE}

CC BY-NC-ND 4.0

\section{REPOSITORY RECORD}

Coates, Tim, and Gary J. Page. 2016. "LES of High Speed Jet Flow from Convergent-divergent Rectangular S-bend Ducts Using Synthetic Inlet Conditions". figshare. https://hdl.handle.net/2134/22503. 


\title{
LES of High Speed Jet Flow From Convergent-Divergent Rectangular S-bend Ducts Using Synthetic Inlet Conditions
}

\author{
Tim D. Coates* and Gary J. Page ${ }^{\dagger}$ \\ Loughborough University, Loughborough, LE11 3TU, United Kingdom
}

\begin{abstract}
The effect of upstream duct curvature on the exhaust plume of a jet engine is further studied. Using synthetically created turbulence, improvements are made to the flow through out the S-bend validation case previously studied. The effect of a contracting $70^{\circ}$ S-bend duct on the over-expanded exhaust plume emanating from a rectangular nozzle of aspect ratio 5.8:1 at a nozzle pressure ratio of 2.5 and Reynolds number of $7.61 \times 10^{5}$ is then studied. A modified version of the synthetic eddy method for creating artificial turbulence is initially validated. The validation of the Hydra CFD code is then expanded upon for an S-bend duct including both RANS and LES methodologiesFor the combined S-bend and nozzle cases the total pressure gradients that were previously observed at the nozzle exit plane for $k-\epsilon$ RANS are also similarly observed using LES with synthetically created inflow turbulence thus confirming the existence of such features. Calculations were carried out using an unstructured, median-dual CFD solver with predominantly hexahedral elements containing approximately 175 million nodes.
\end{abstract}

\section{Introduction}

Future civil and military applications may depart from the typical design of propulsive gas turbine installations. These future applications, such as the renewed interest in propfan or 'open rotor' engines, may include the coupling of a convoluted duct to a non-circular nozzle. Prior to the work of Coates and Page ${ }^{1}$ the contributions made by the research community regarding an S-bend duct has focused on inlet applications, typically the suppression of separation and secondary flows.

The RANS calculations of an S-bend duct coupled to a high aspect ratio nozzle ${ }^{1}$ displayed a transverse total pressure gradient at the nozzle throat. The transverse total pressure gradient was notably different in the equivalent LES calculations and was attributed to a poorly resolved upstream flow. It was thought that, in order to improve the upstream flow, the representation of turbulence at the inlet plane required improvement. This paper continues the work of Coates and Page, ${ }^{1}$ improving the modelling of the upstream, convoluted duct flow through the use of LES and synthetic inlet conditions.

The formation of a secondary flow in an S-bend, as described by Miller ${ }^{2}$ and Prandtl, ${ }^{3}$ occurs due to a combination of adverse pressure gradients and centrifugal forces acting on the flow. At the start of the bend the flow is deflected to the outside of the duct as the pressure increases in the direction of the flow. This decelerates the boundary layer and in turn causes friction within the fluid further slowing the boundary layer. If the velocity decays sufficiently quickly then the flow separates from the wall and a secondary flow occurs. This secondary flow is a reverse flow formed as the low speed fluid moves around the walls towards the inside of the bend where there is a low static pressure region. This initialises the two vortices which can be seen in S-shaped ducts such as those encountered by Bansod and Bradshaw. ${ }^{4}$

The development of the flow in a square cross-sectioned, S-shaped duct was investigated by $\mathrm{Ng}$ et al. ${ }^{5}$ and Taylor et al. ${ }^{6}$ Flow separation was seen to occur along the lower wall of the first bend as well as along

${ }^{*}$ Ph.D. Student, Dept. of Aeronautical and Automotive Engineering. Email: t.coates@lboro.ac.uk, Tel: +44 (0) 1509227282

†Senior Lecturer, Dept. of Aeronautical and Automotive Engineering, AIAA Senior Member. Email: g.j.page@lboro.ac.uk, Tel: $+44(0) 1509227205$ 
the outer wall of the second bend by both groups of authors. However the secondary flow caused by the first bend is weakened throughout in the second bend due to the opposing swirl created by the secondary flow along the outer wall. Further research has also been conducted by Vakili et al., ${ }^{7-10}$ Smith et al. ${ }^{11,12}$ and Guo and Seddon. ${ }^{13,14}$

Early LES calculations focused predominantly on flows that lent themselves to periodic boundary conditions in the streamwise direction (such as channel and pipe flows). By recycling the flow field at the outlet plane, realistic turbulence can be modelled modelled on the inlet plane. However, due to the increase in computing power more complex problems are being studied. Whilst calculations involving low freestream turbulence do not require complete, realistic, inlet data, those that include spatially developing flows do. Studies performed by Klein et al. ${ }^{15}$ Lund et al. ${ }^{16}$ and Jarrin et al. have all shown that the upstream inflow conditions have a direct impact on the accuracy of the flow. Consequently the use of artificially created inflow turbulence has been the subject of much interest in the recent years. Keating et al. ${ }^{17}$ gives an overview of the various approaches to artificially creating inflow turbulence. The four main approaches for creating artificial inflow turbulence, as stated by Keating et al., ${ }^{17}$ are outlined below.

The simplest form of creating realistic turbulence at the inlet is via the use of a precursor simulation. This involves performing an LES calculation on a secondary mesh prior to the main simulation. The secondary mesh has a a cross-section matching the inlet plane of the simulation and a length sufficient for the growth of a turbulent boundary layer to occur. The fluctuating turbulent boundary layer can then be written to a file or fed directly into the inlet plane of the main simulation. This approach can be very costly due to the length of duct required for the boundary layer to grow. The turbulent inlet conditions may also require rescaling in order order to match experimental or theoretical conditions and the overall method copes poorly with non-uniform flows.

Lund et al. ${ }^{16}$ used a variation of the precursor simulation to create three dimensional, time dependent, turbulent inflow data. The method suggested by Lund et al. involves coupling a secondary simulation to the initial problem. This secondary simulation is based on the inlet plane geometry and uses periodic boundary conditions to create the turbulent boundary layer without the need to have a lengthy development section. To achieve the desired properties of the flow, such as boundary layer thickness, the periodic outflow is rescaled to match these properties. This recycling and rescaling method gives very similar results to those found by the direct simulation of a turbulent boundary layer by Spalart. ${ }^{18}$ However, this method has similar shortcomings to the precursor simulation method and would also be difficult to implement in an unstructured solver.

Klein et al. ${ }^{15}$ developed a method of using digital filters to generate artificial inflow turbulence. Using known, or estimated, statistical properties a velocity signal is generated on which digital filters are applied to obtain turbulence with two-point correlation. Mare et al. ${ }^{19}$ expanded the method to incorporate the reproduction of multidimensional space-time correlations, a feature which is more desirable in wall bounded flows.

Using artificially created eddies, Jarrin et al. ${ }^{20}$ proposed the Synthetic Eddy Method (SEM) for the generation of turbulent inflow data. The SEM provided both temporally and spatially correlated data using a Gaussian function to give the 'shape' of the eddies. The three dimensional expansion of the SEM outlined by Jarrin et al. ${ }^{21}$ appears to yield a reasonable approximation to realistic turbulence; however, the directional components of the eddies are limited to eight orthogonal vectors, where $u_{i}= \pm 1$. This reduces the random component of the intensity of the eddies thus reducing the realism of the synthetic turbulence. It was also noted that the velocity distribution function, $f_{\sigma}$, is dimensionally inconsistent in the work of Jarrin et al., ${ }^{21}$ presumably introducing some errors into the calculation.

Using a modified version of the Synthetic Eddy Method (SEM) created by Jarrin et al. ${ }^{20,21}$ we aim to improve the flow modelling of the various convergent S-bends seen in part 1 . We will then use this improved flow modelling to further investigate the effects of upstream duct convolution with a converging S-duct, on the jet plume emanating from a convergent-divergent high aspect ratio nozzle. Initially the SEM implimentation will be discussed in section II with a validation of the method shown in section III. The subsequent study into the use of the SEM inlet conditions for the three different curvature unconventional aeroengine exhaust systems used by Coates and Page ${ }^{1}$ is then evaluated in section IV. 


\section{Methodology}

This study uses the Rolls-Royce solver, Hydra. ${ }^{22}$ Hydra is a density-based solver for compressible flows on unstructured grids. This allows the code to simulate flows of complex geometries, such as severe curvature S-bend ducts, easily. The Rolls-Royce solver and general CFD methodology is outlined in more detail by Coates and Page. ${ }^{1}$ A modified version of the Synthetic Eddy Method (SEM) by Jarrin et al., ${ }^{20,21}$ outlined below, has been implemented into the Hydra solver.

\section{II.A. Synthetic Eddy Method}

The finite set of points $S=\left\{\boldsymbol{x}_{1}, \boldsymbol{x}_{2}, \ldots, \boldsymbol{x}_{s}\right\}$ represents the inlet plane on which we want the SEM to generate synthetic velocity fluctuations. Initially, a virtual box containing the synthetic eddies is created surrounding the inlet plane. The dimensions of the virtual box are defined by the inlet plane and the length scale of flow, $\sigma$, such that

$$
\sigma=\max \left\{\min \left\{\frac{k^{\frac{3}{2}}}{\epsilon}, \kappa \partial\right\}, \Delta\right\}
$$

where $\kappa=0.41, \Delta=\max (\Delta x, \Delta y, \Delta z)$ and $\partial$ is the thickness of the desired boundary layer. The coordinates of the virtual box are then defined by

$$
x_{i, \min }=\min _{x \in S}\left(x_{i}-\sigma(\boldsymbol{x})\right) \quad \text { and } \quad x_{i, \max }=\max _{x \in S}\left(x_{i}+\sigma(\boldsymbol{x})\right) .
$$

Within the virtual box, the number of synthetic eddies created is kept at a constant value, $N$, throughout the simulation. This number depends on the volume of the virtual box, $V_{b}$ and the length scale, $\sigma$, such that $N=\max \left(V_{b} / \sigma_{i}\right)$. The velocity fluctuations at a point $\boldsymbol{x}_{i}$ on the inlet plane can then be calculated using $u_{i}=U_{i}+u_{i}^{\prime}$ where $U_{i}$ is the mean velocity flow at point $\boldsymbol{x}_{i}$ and $u_{i}^{\prime}$ is the synthetic velocity fluctuation caused by the eddies acting on the point $\boldsymbol{x}_{i}$. The synthetic velocity fluctuation, $u_{i}^{\prime}$, is defined by

$$
u_{i}^{\prime}=\frac{1}{\sqrt{N}} \sum_{k=1}^{N} a_{i} j \epsilon_{j}^{k} f_{\sigma x}\left(\boldsymbol{x}-\boldsymbol{x}^{k}\right)
$$

where $\epsilon_{j}^{k}$ is the intensity of the eddy located at $\boldsymbol{x}^{k}, f_{\sigma \boldsymbol{x}}\left(\boldsymbol{x}-\boldsymbol{x}^{k}\right)$ is the velocity distribution of an eddy located at $\boldsymbol{x}^{k}$. The magnitude of the velocity fluctuation is determined by the Cholesky decomposition of the Reynolds Stress tensor, $a_{i j}$.

Similarly to the work of Jarrin et al. ${ }^{21}$ the shape of the synthetic eddies were defined by a simple tent function,

$$
f(x)= \begin{cases}\sqrt{\frac{3}{2}}(1-|x|) & \text { if }|x|<1 \\ 0 & \text { otherwise. }\end{cases}
$$

This, combined with the assumption that the effects of a synthetic eddy only has an impact on a point on the inlet plane within one $\sigma$ of it's location, gives the velocity distribution of an eddy located at $\boldsymbol{x}^{k}$ as

$$
f_{\sigma x}\left(\boldsymbol{x}-\boldsymbol{x}^{k}\right)=\sqrt{\frac{V b}{\sigma^{3}}} f\left(\frac{x-x^{k}}{\sigma}\right) f\left(\frac{y-y^{k}}{\sigma}\right) f\left(\frac{z-z^{k}}{\sigma}\right) .
$$

The locations of the synthetic eddies, $\boldsymbol{x}^{k}$, are randomly distributed using a uniform distribution over the virtual box defined by equation 1 . The corresponding intensities, $\epsilon_{j}^{k}$, are randomly taken over the range $[-\sqrt{3}, \sqrt{3}]$.

Through the use of a constant velocity, $\boldsymbol{U}_{c}$, characteristic of the flow the synthetic eddies achieve a temporal coherence as they are convected through the virtual box. After each iteration with timestep $d t$, the new position of eddy $k$ is given by

$$
\boldsymbol{x}^{k}(t+d t)=\boldsymbol{x}^{k}(t)+\boldsymbol{U}_{c} d t .
$$




\section{II.B. Parallel Processing}

The Hydra solver uses the MPI and OPlus libraries for parallel communication and the grid is partitioned at run time using ParMetis. A separate copy of the SEM inlet condition generator is run on each processor due to the paralization of the calculation.

The LES cases were run for 100, 000 timesteps before the mean was turned on. The mean was measured across the next 500,000 timesteps sampling every 10 timesteps. The S-bend duct simulation using the SEM implementation took approximately 53 hours over 640 processors on the Midlands high performance computer, Hera. This was around Y\% longer than a similarly run simulation without the SEM.

\section{Validation Study}

Similarly to the Hydra code RANS validation study of convoluted ducts by Coates and Page ${ }^{1}$ the work of $\mathrm{Ng}$ et al. ${ }^{5}$ will be used to assess both validity of the SEM implementation within Hydra as outlined in section II and the the viability of the Hydra LES code, both with and without the SEM implementation.

The third test section from the $\mathrm{Ng}$ et al. ${ }^{5}$ experiments, shown in figure 1 , will be used again in the validation study due to the large offset and severe curvature of the geometry.

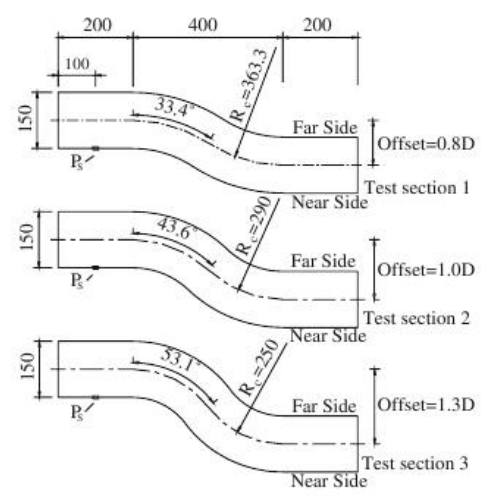

Figure 1: Schematic drawing of the three test sections used by Ng et al.

\section{III.A. The Geometry and Mesh of the S-bend Duct, Test Section 3}

The experimental model consisted of the $0.8 \mathrm{~m}$ test section connected to a $1 \mathrm{~m}$ long, $12: 1$ contraction at the inflow end of the test section and a $2 \mathrm{~m}$ diffusing duct at the outflow end. The system was driven by an open loop suction wind tunnel which is shown in figure 2. Two meshes were used in the validation study; both created using the ANSYS ICEM unstructured block mesh generator.

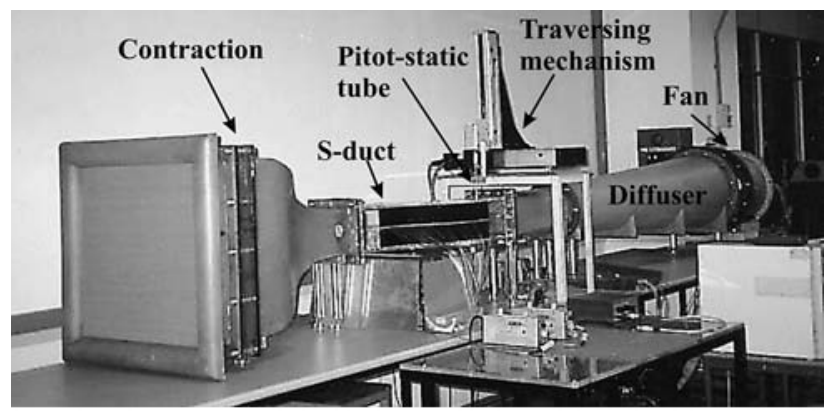

Figure 2: The experimental set-up used by Ng et al. 
Test section 3 has a hydraulic diameter of $0.15 \mathrm{~m}$ and is split into three components. Either end of the test section section is made up of a straight duct, each $0.2 \mathrm{~m}$ long. The S-bend takes up the remaining $0.4 \mathrm{~m}$ of the test section and has a radius of curvature of $0.25 \mathrm{~m}$ and an angle of curvature of $53.1^{\circ}$ giving an offset of $1.3 D_{h}$. The first of the two straight ducts also contains the reference static pressure tap $\left(P_{s}\right)$ which was located in the centre of the lower wall of the duct as is highlighted in figure 1.

Figure 3 displays the mesh used in the RANS validation study with the inlet shown in yellow, the contraction in red and the test section in blue. The diffuser and outlet are then coloured purple and green respectively. The RANS mesh of the experiment is comprised of approximately 9.2 million nodes. The test section, shown in figure $3 \mathrm{~b}$ has approximately 4.1 million nodes with the cross-sectional slice being 101 square nodes.

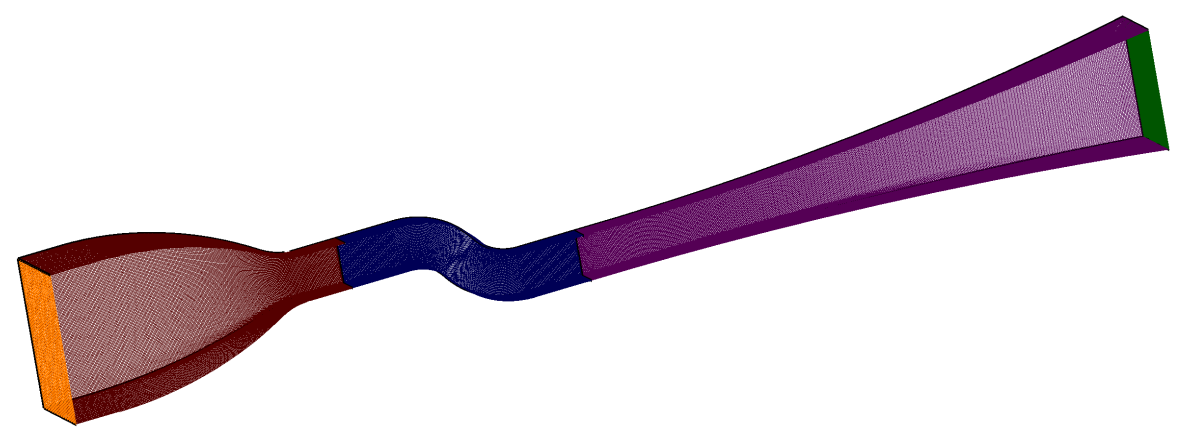

(a) Overall mesh

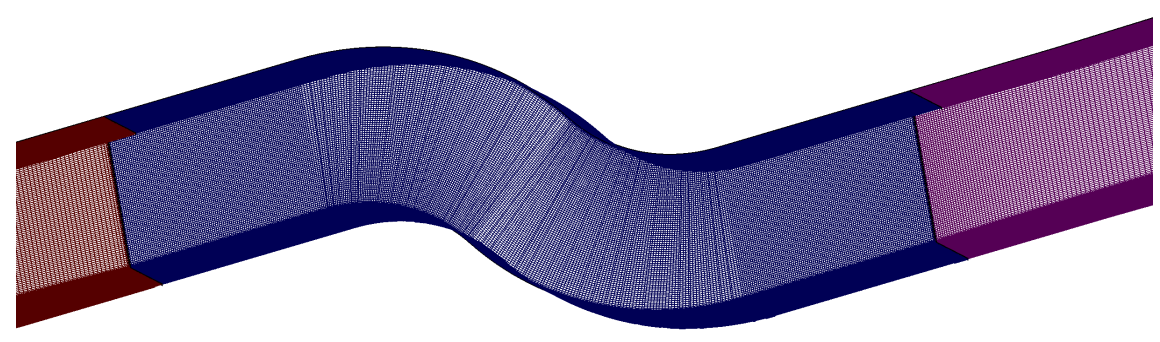

(b) S-bend region of Mesh

Figure 3: The NGSD-3 S-bend RANS mesh.

The mesh used for the LES and SEM validation studies is shown in figure 4 and was comprised of 10.5 million nodes. This second mesh is limited to just the test section and a short, $0.2 \mathrm{~m}$, section of the outlet region of the outlet section to ensure that the outlet plane does not interfere with the data collected from the end of the test section. The cross section of the duct consists of X nodes with $z^{+}=1, y^{+}=2.6$ and $x^{+}=2.6$. 


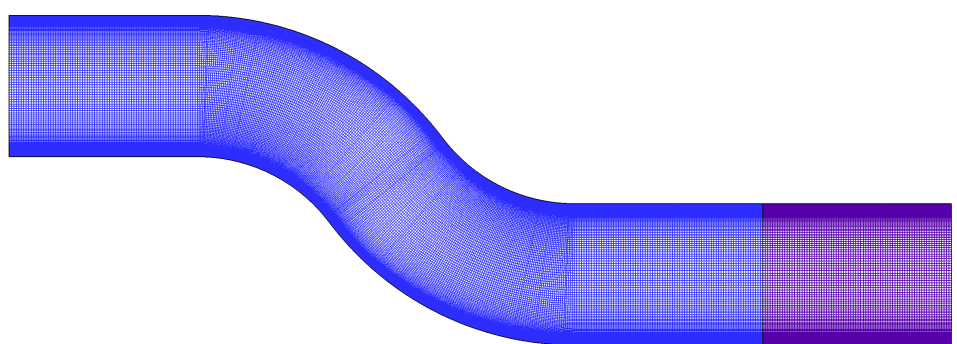

(a) The overall mesh

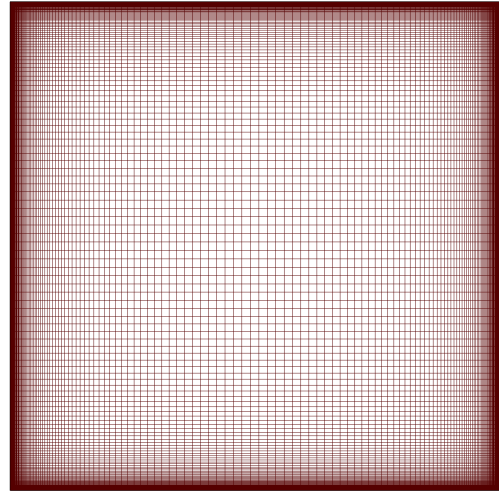

(b) A cross section of the mesh

Figure 4: The NGSD-3 S-bend LES mesh.

\section{III.B. SEM Validation}

The SEM implementation is validated both as a stand-alone version of the code and as part of the Hydra CFD code. In order to generate the initial Reynolds stresses used to calibrate the SEM a precursor RANS simulation using the FLUENT Reynolds-Stress second order turbulence model was conducted. The geometry used in the precursor simulation has the same cross section as the $\mathrm{Ng}$ et al. S-bend duct and is $0.2 \mathrm{~m}$ long with a corresponding uniform mesh of $50 \times 50 \times 50$ nodes.

Figure 5 shows a comparison of the Reynolds stresses at the inlet plane between the data used to initialise the SEM, the stand-alone version of the SEM and the SEM implemented in Hydra using the LES S-bend mesh. The Stand-alone version was run for two million timesteps with a sample taken for the mean every 100 timesteps. The stand-alone SEM produces a very near match to the initialising data proving that the SEM does not introduce a bias into the flow due to the eddies. The Hydra implementation of the SEM does appear to have a similar, though slightly damped approximation to the initialization data. This damping effect may well be due to the Reimann invariance built into the CFD code for XXX. 


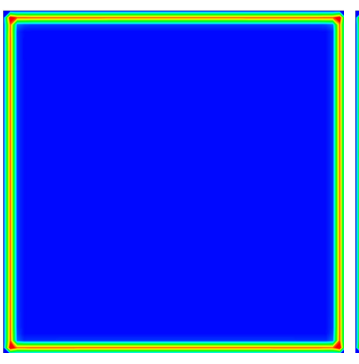

(a) Baseline $R_{u u} / U_{i}{ }^{2}$

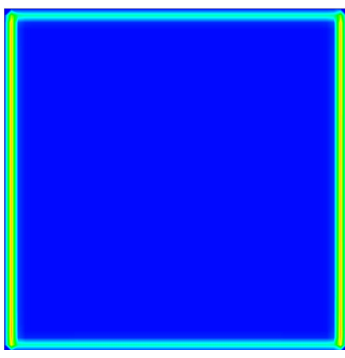

(e) Baseline $R_{v v} / U_{i}{ }^{2}$

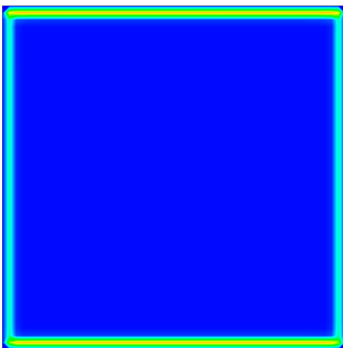

(i) Baseline $R_{w w} / U_{i}{ }^{2}$

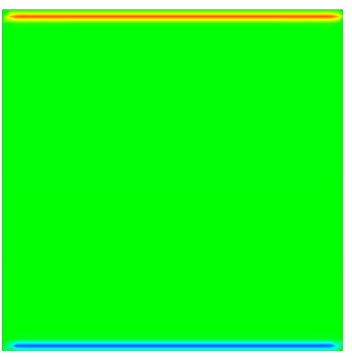

(m) Baseline $R_{u v} / U_{i}{ }^{2}$

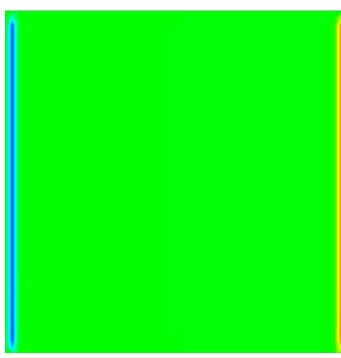

(q) Baseline $R_{w u} / U_{i}^{2}$

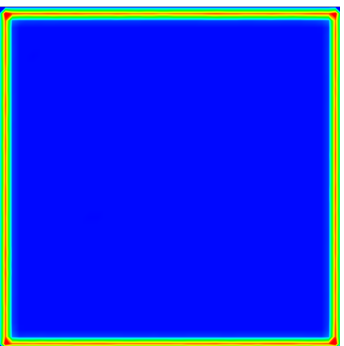

(b) Stand-Alone SEM

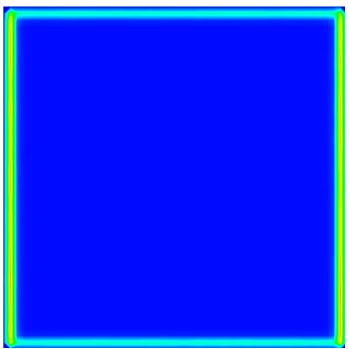

(f) Stand-Alone SEM

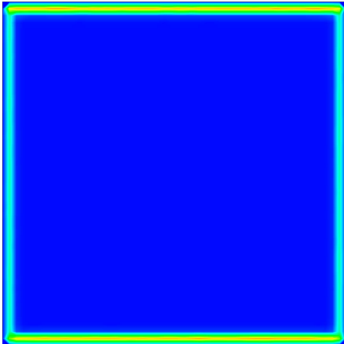

(j) Stand-Alone SEM

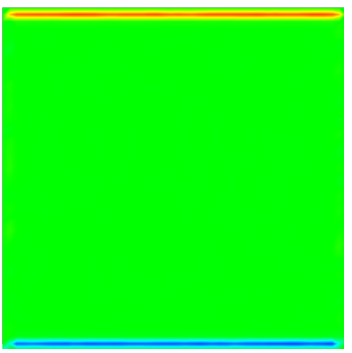

(n) Stand-Alone SEM

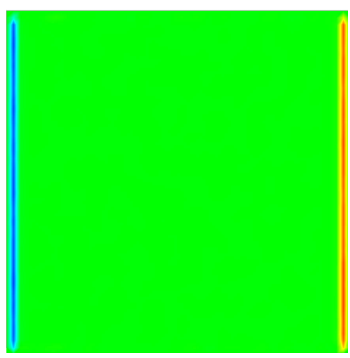

(r) Stand-Alone SEM

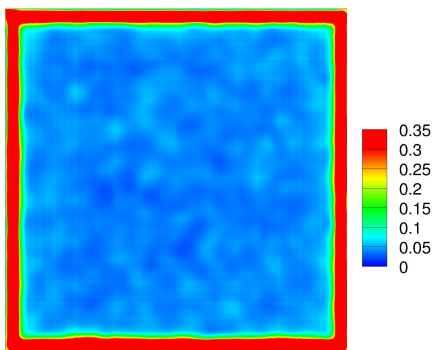

(c) Hydra-SEM

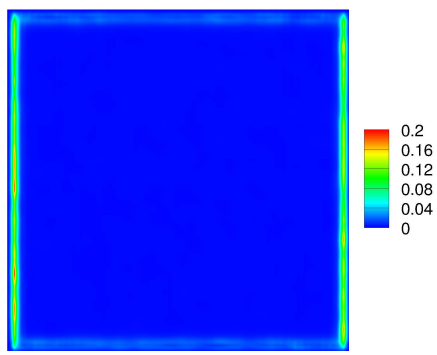

(g) Hydra-SEM

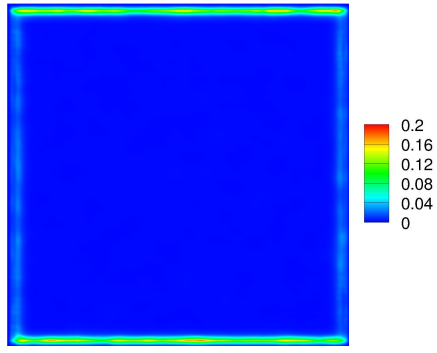

(k) Hydra-SEM

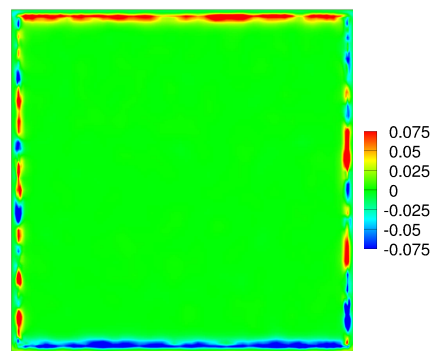

(o) Hydra-SEM

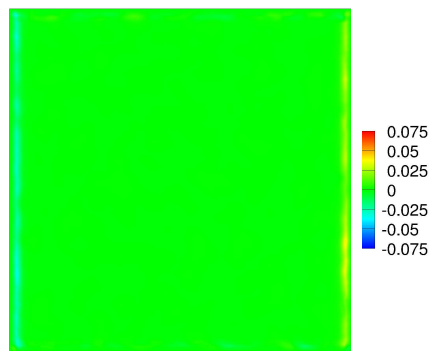

(s) Hydra-SEM

Figure 5: Baseline Reynolds stresses on the inlet plane compared against calculated Reynolds stresses from the stand-alone SEM code and the LES Hydra implementation of the SEM. 


\section{III.C. Comparison of CFD Methods for an S-bend Duct of $1.3 D_{h}$ offset.}

The comparison of the RANS simulations for the $\mathrm{Ng}$ et al. S-bend duct were outlined in the work of Coates and Page. ${ }^{1}$ The simulations were run using four different turbulence models. The Rolls Royce Hydra code (version 6.2.16) was used for the Spalart-Allmaras, $k-\epsilon$ and $k-\omega$ SST turbulence models whilst the ANSYS Fluent code was used for the Reynolds-Stress second order turbulence model. The simulation took approximately 7500 iterations in order to achieve a rough level of convergence with the vortices stabilising after 17500 iterations.

Throughout the literature ${ }^{6,23}$ it is well known that the pressure difference between the near and far walls changes sign due to the effects of the duct curvature. Similarly this behaviour can be seen along the centreline of the RANS simulations as shown in figure 6. All four turbulence models exhibit the same behaviour in that the flow through the first bend matches very closely with the experimental data. However, throughout the second the bend the simulations under predict the pressure recovery seen in the experimental data. The inflection point, denoting the distortion in the pressure coefficient caused by the flow separation, is also substantially weaker than expected and occurs further downstream.

The centreline static pressure distributions for the large eddy simulations are shown in figure 7 along with the Spalart Allmaras RANS turbulence model for comparison. Here we can see that there is a stark difference between the two SGS models. The Smagorinsky SGS displays a similar under prediction of pressure recovery throughout the second bend whilst the WALE SGS matches considerably closer to the experimental data. Both SGS models also display the inflection point, however both show a delayed start to the point of flow separation.

In the first bend there appears to be a laminar flow separation in the two SGS models. However, with the inclusion of the synthetic inlet conditions the laminar flow separation is eliminated from the WALE SGS model and the inflection point is also brought approximately in line with the experiment. These laminar flow separations, seen in figure 8 , are due to the lack of turbulent fluctuations within the flow. Their absence from the non SEM cases is seen clearly in the Q-criterion visualisations of the start of the test section; shown in figure ??.

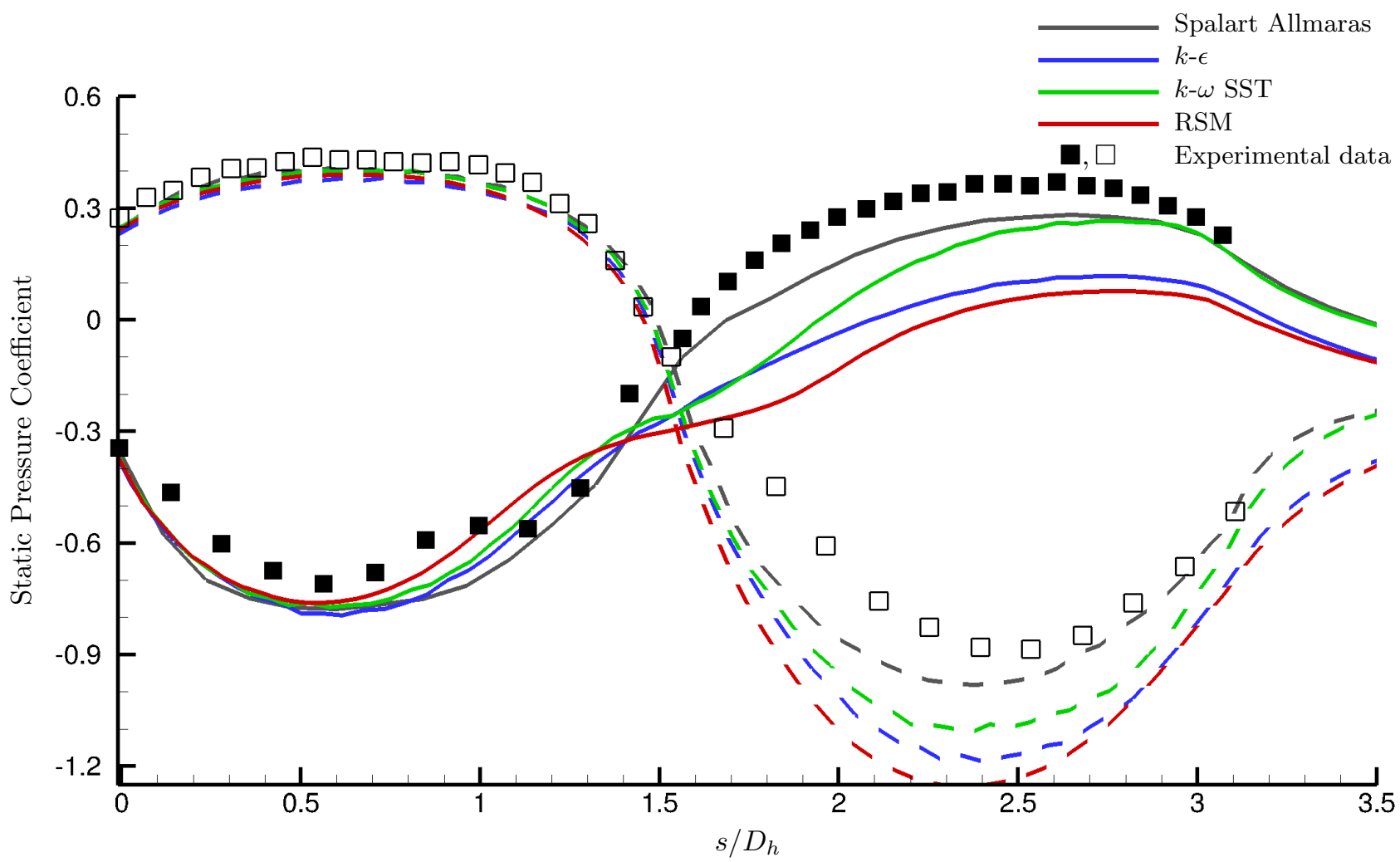

Figure 6: The Centreline static pressure distribution for RANS turbulence models. The solid line and the dashed line represent the lower wall and upper wall resepctively. 


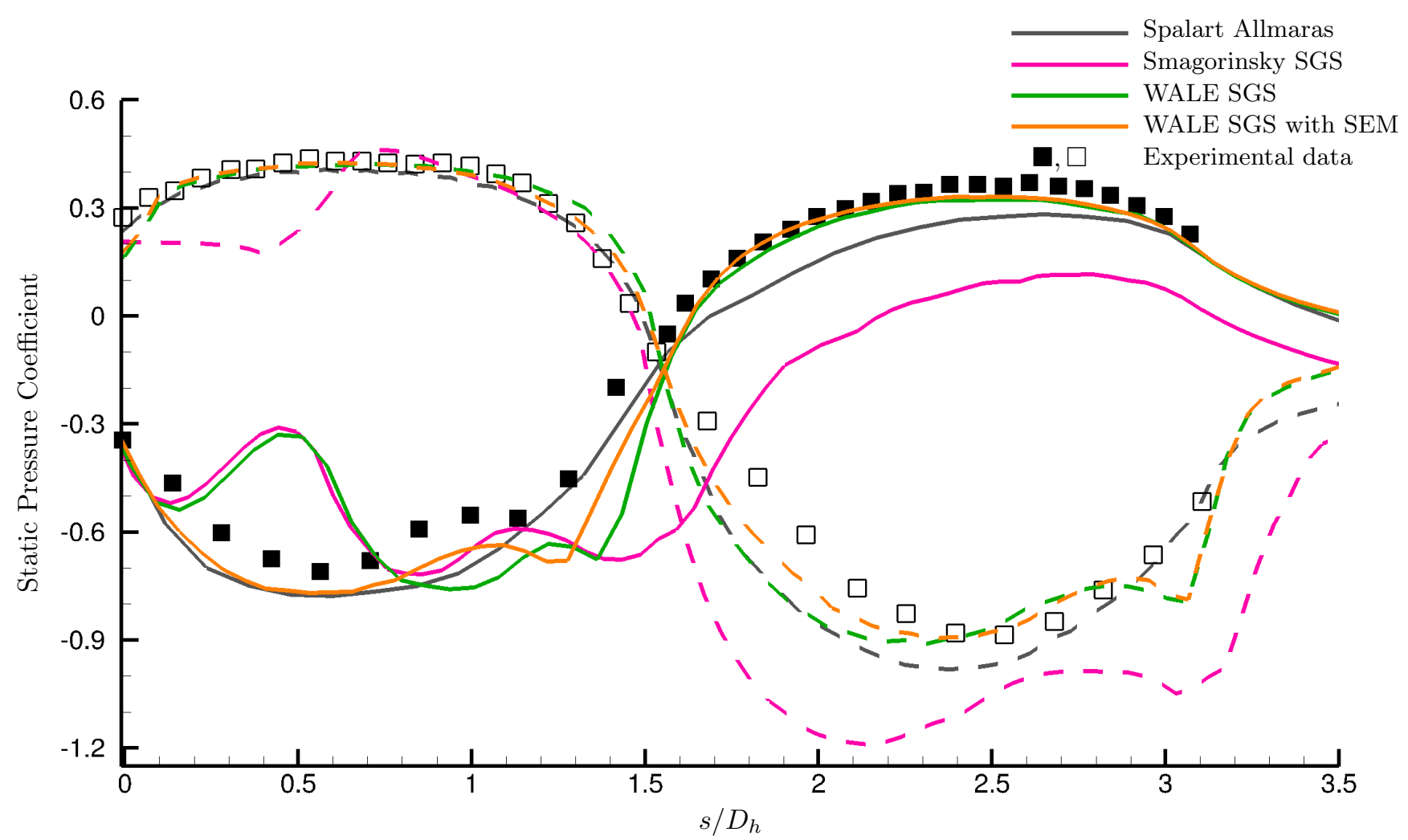

Figure 7: The Centreline static pressure distribution for large eddy simulations. The solid line and the dashed line represent the lower wall and upper wall resepctively.

Figure 9 describes the total pressure contours for the experimental data, Spalart-Allmaras and $k-\omega$ SST RANS cases as well as the three LES cases for test section 3. Both RANS turbulence models show two distinct low pressure regions on the left side of the subfigures, caused by the streamwise vortices. This is similarly seen for the Smagorinsky model???? however the WALE SGS model shows both a merged layer of lower total and the two distinct regions of low total pressure in each corner. The SEM implementation of the WALE model shows a similar merged low pressure region with even less evidence of the twin cores in the total pressure distribution.

Similarly to the total pressure contours, figure 10 compares the crossflow velocity of the CFD simulations against the experimental data collected by $\mathrm{Ng}$ et al. of test section 3 . In each subfigure showing the CFD simulations a red star is used to pin-point the centre of the vortices seen in the experimental data.

Two opposing vortices are observed in each half of the experimental duct as shown in figure 10a. The interaction between the two vortical structures causes the separation between the primary and secondary flows as seen by $\mathrm{Ng}$ et al. For both RANS models an extra vortex occurs nearer to the centreline of the duct in both halves. This is similarly seen in the LES SGS models as well however in the case of the SEM implementation, the positions of two of the vortices present match very closely with the experimental data. 


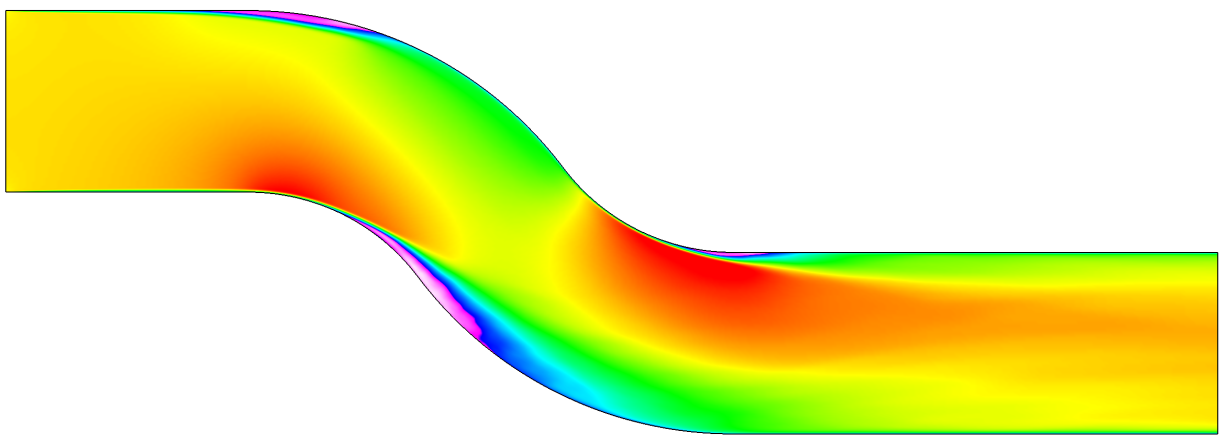

(a) RANS, $k$ - $\omega$ SST Turbulence Model - PLACE HOLDER

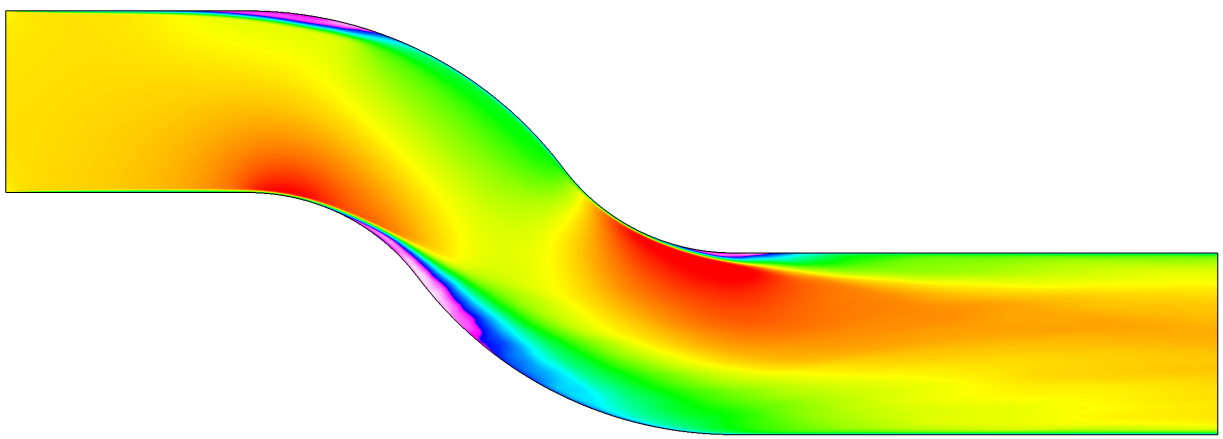

(b) Smagorinsky SGS

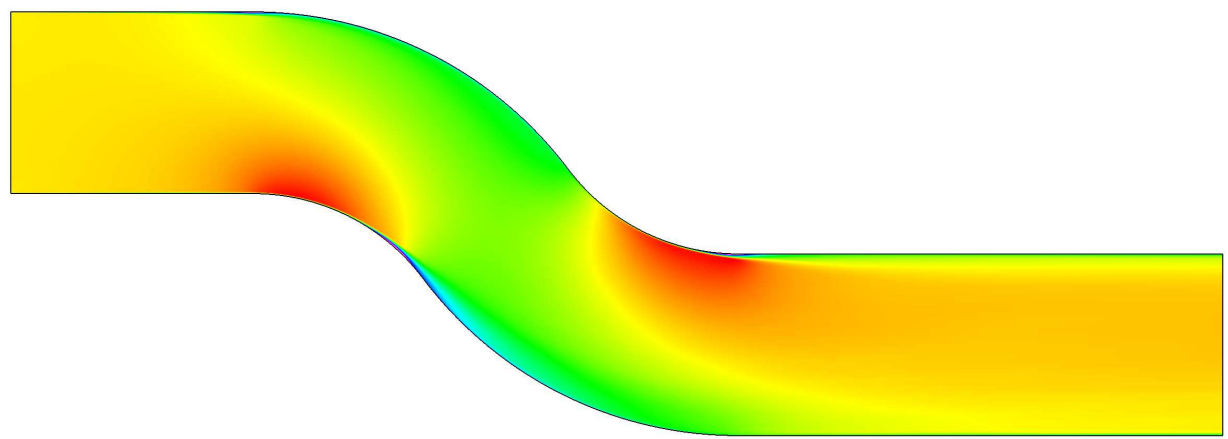

(c) Wale SGS

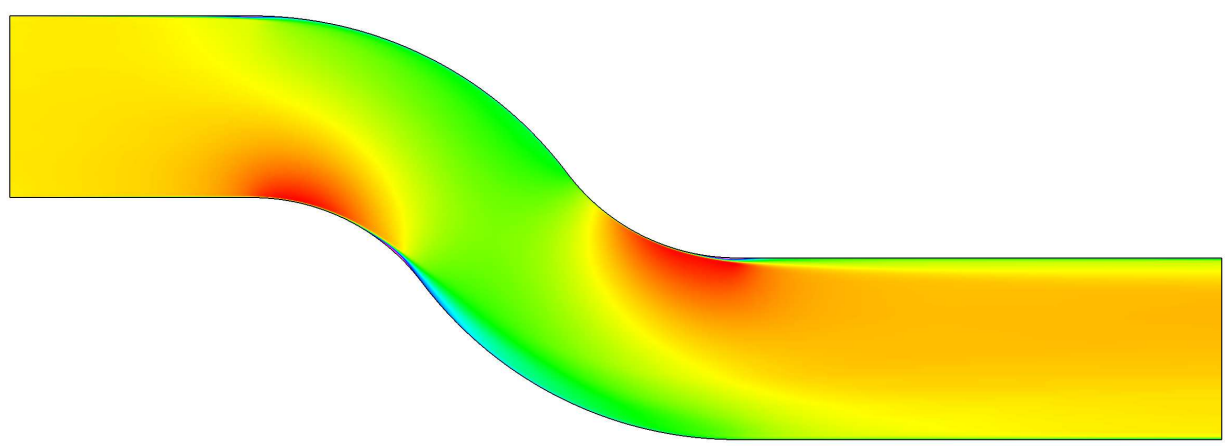

(d) Wale SGS with synthetic inlet conditions

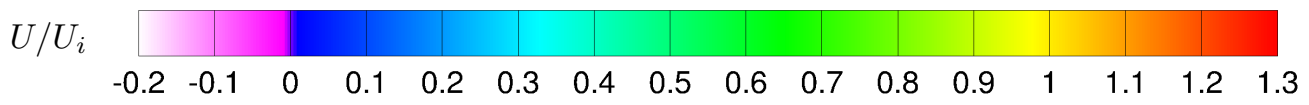

Figure 8: The normalised streamwise velocity distribution across the centreplane of the S-duct.

\section{0 of 16}




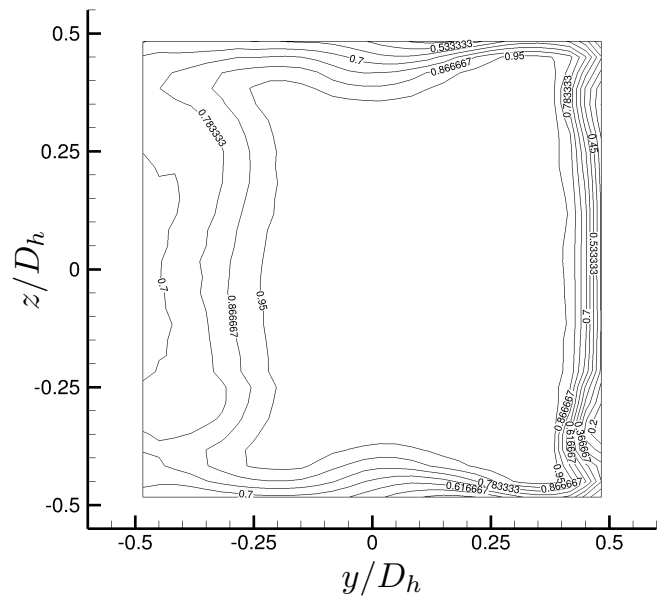

(a) Experimental data $1.3 D_{h}$ offset

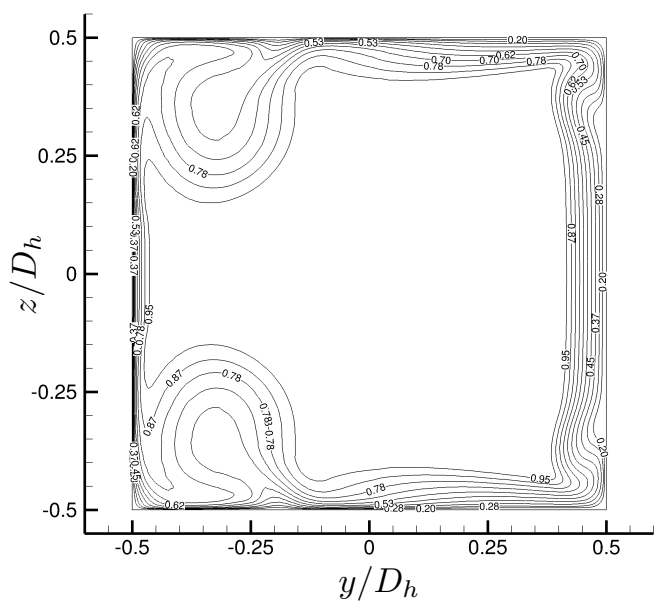

(c) Spallart Almaras turbulence model

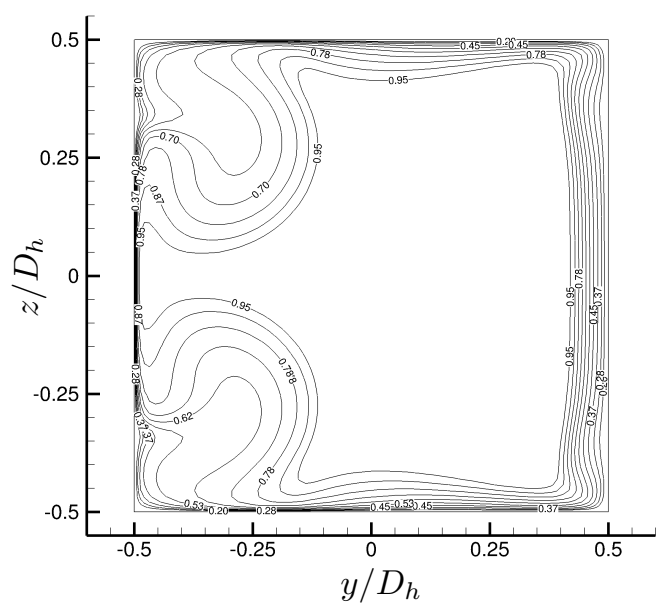

(e) $k-\omega$ SST turbulence model

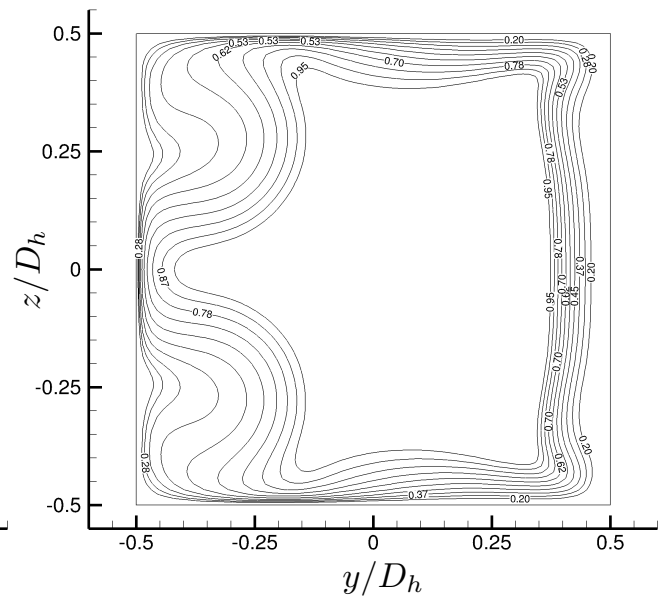

(b) Smagorinsky SGS model

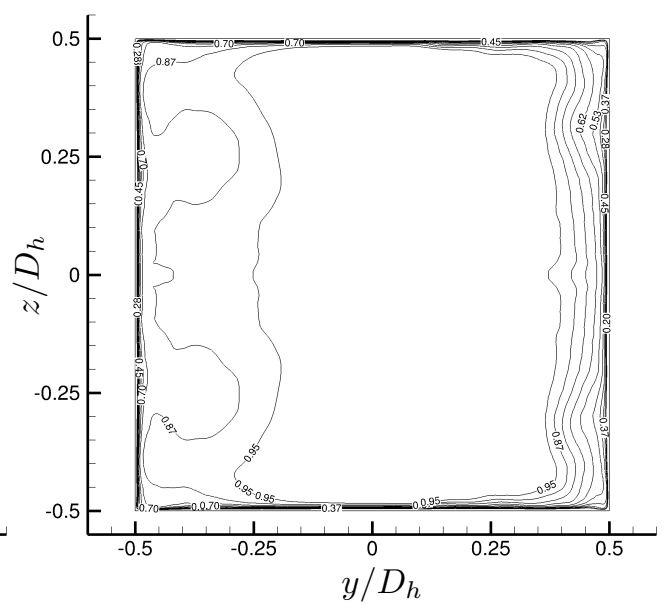

(d) WALE SGS model

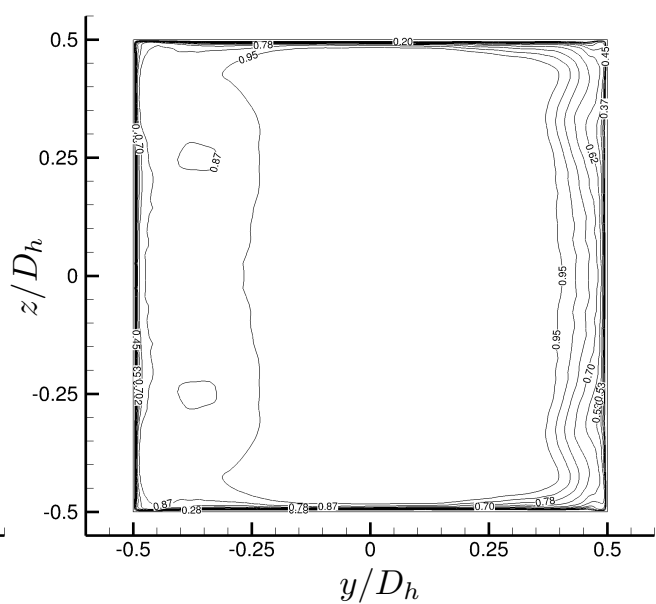

(f) WALE SGS model using the SEM

Figure 9: Total pressure contours at the test section outlet. 


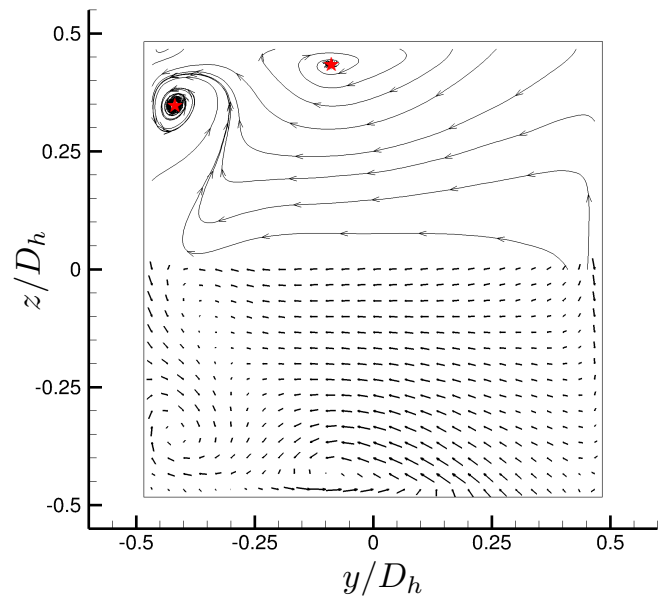

(a) Experimental data

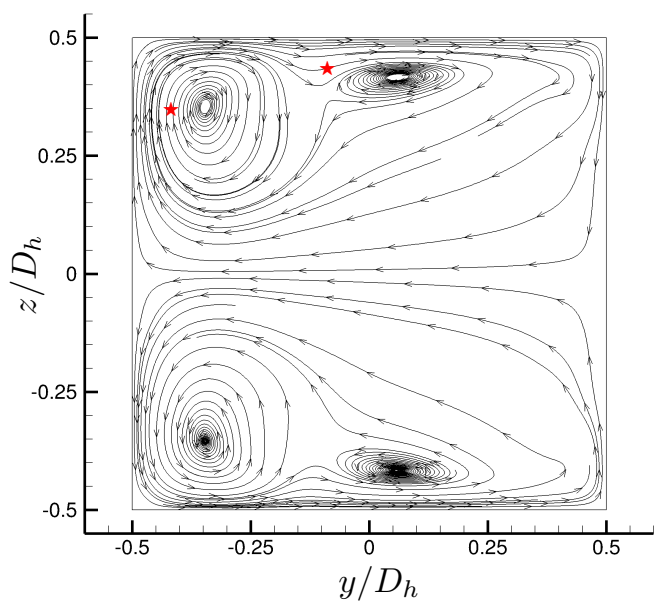

(c) Spallart Almaras turbulence model

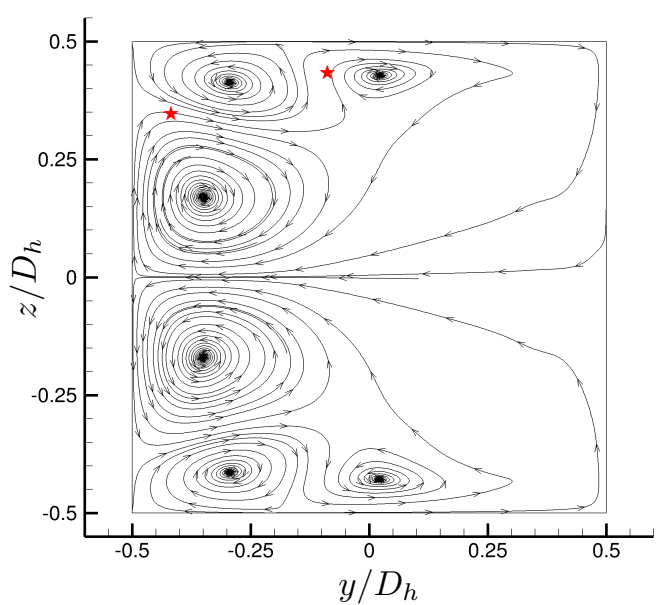

(e) $k-\omega$ SST turbulence model

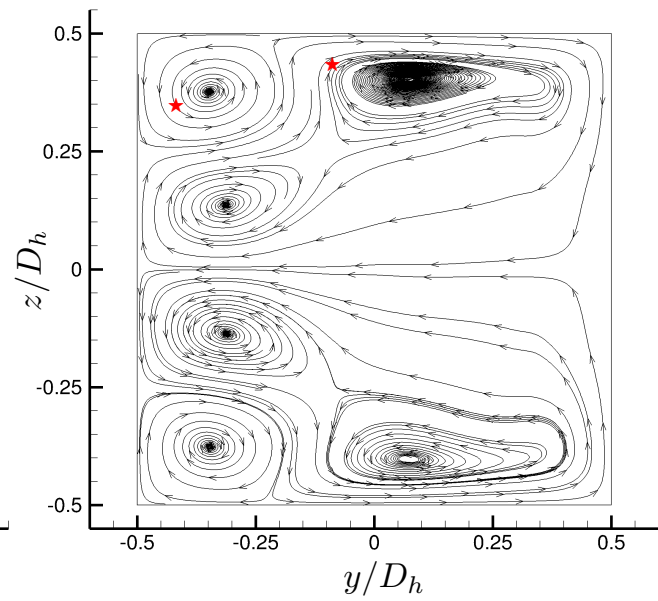

(b) Smagorinsky SGS model

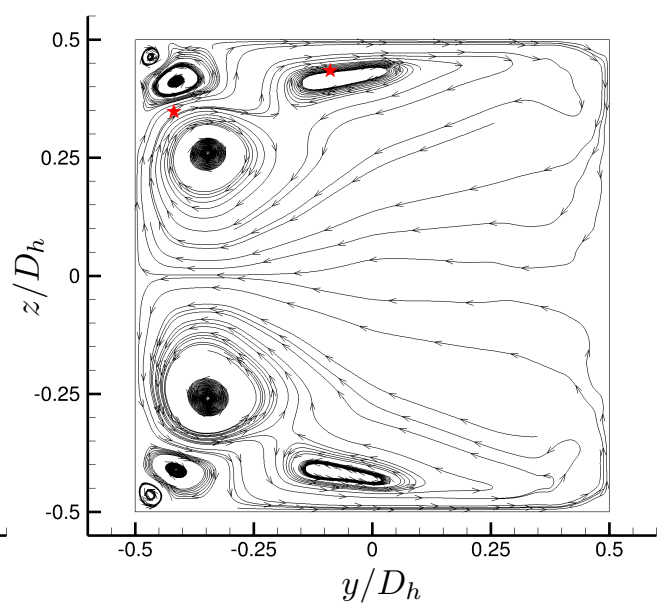

(d) LES with WALE SGS model

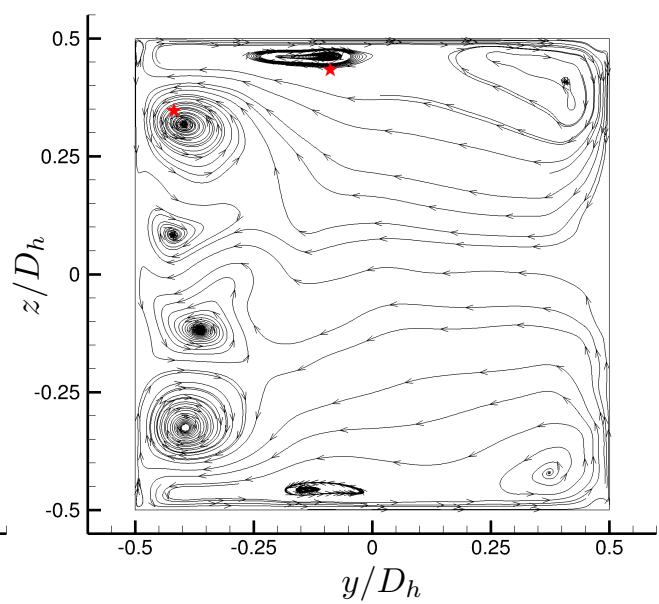

(f) LES with WALE SGS model

Figure 10: Crossflow velocity at the test section outlet. The red stars in figures 10c-10f denote the center of the vortices seen in the experimental data for test section 3. 


\section{CFD Comparison of Converging S-ducts attached to the RRLU1 Divergent Nozzle}

The unconventional aeroengine exhaust system (UAES), as described by Coates and Page, ${ }^{1}$ combines a contracting S-bend duct with a high aspect ratio divergent nozzle. Three different S-duct curvatures (SDC) were originally considered, for the purposes of investigating the effects and potential improvements made by the inclusion of the SEM to the Hydra CFD code only the $70^{\circ}$ curvature case is considered.

\section{IV.A. Geometry}

The geometry for the UAES is shown in figure 11. The inlet section is $97.62 \mathrm{~mm}$ long, with a rectangular cross-section $91.68 \mathrm{~mm} \times 25 \mathrm{~mm}$, this is followed by the $70^{\circ}$ contracting S-bend test section with a $1.09 D_{h}$ offset. The divergent section of the nozzle comprises of a $55.78 \mathrm{~mm}$ duct with an increase in duct height of $16.2 \%$ to $15.74 \mathrm{~mm}$. The final element of the geometry is the coflow domain, starting at the throat and continuing $15 D_{h}$ after the nozzle exit. This has a radius of $4 D_{h}$ at the coflow inlet and a radius of $5 D_{h}$ at the exit.

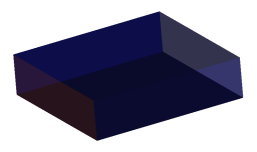

Inlet duct

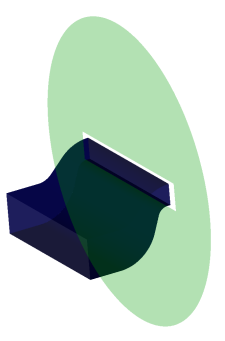

$70^{\circ} \mathrm{SDC}$

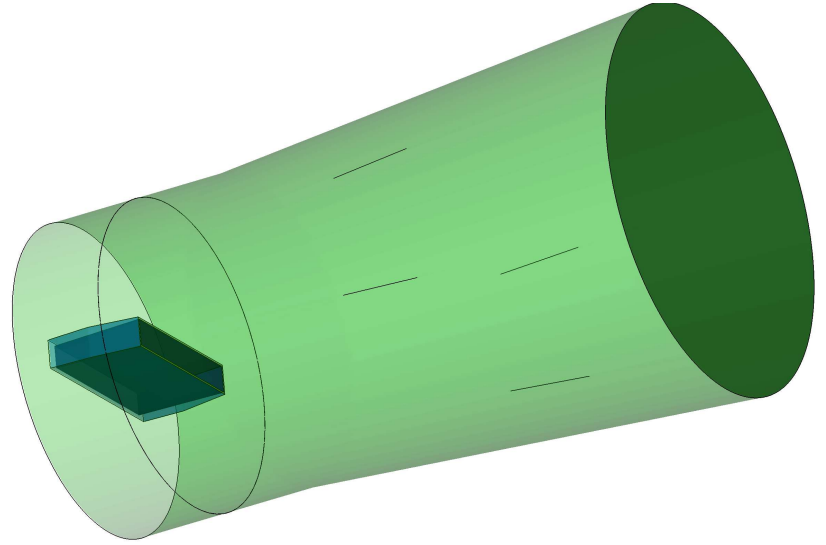

Nozzle and coflow domain

Figure 11: Geometry of the $70^{\circ}$ UAES nozzle.

\section{IV.B. $70^{\circ}$ Comparison}

Although the Spalart-Allmaras turbulence model displayed the best approximation to the experimental results of the S-bend for the RANS validation it was neglected in favour of the $k$ - $\epsilon$ turbulence model due to concerns over the appropriateness for high-speed jet flows.

In the previous study ${ }^{1}$ the $k$ - $\epsilon$ RANS simulation was then compared to the results of the WALE LES. In the same manor we will compare the two previous simulations with that of the SEM implantation of Hydra using the WALE SGS model. Figure 12 shows the minor axis cross section of streamwise velocity. Here it can be seen that both the WALE SGS simulations have a reduced potential core length as expected however the SEM has an upward deflection to the potential core as seen in the $k-\epsilon$ RANS simulation.

The percentage total pressure of several axial stations are then seen in figure 13. The transverse pressure gradient previously seen only in the $k-\epsilon$ RANS simulations once more appears in the SEM implantation of Hydra using the WALE SGS model. This gives a reasonable likelihood that the transverse pressure gradient actually occurs and has a considerable impact on the development of the jet plume. 


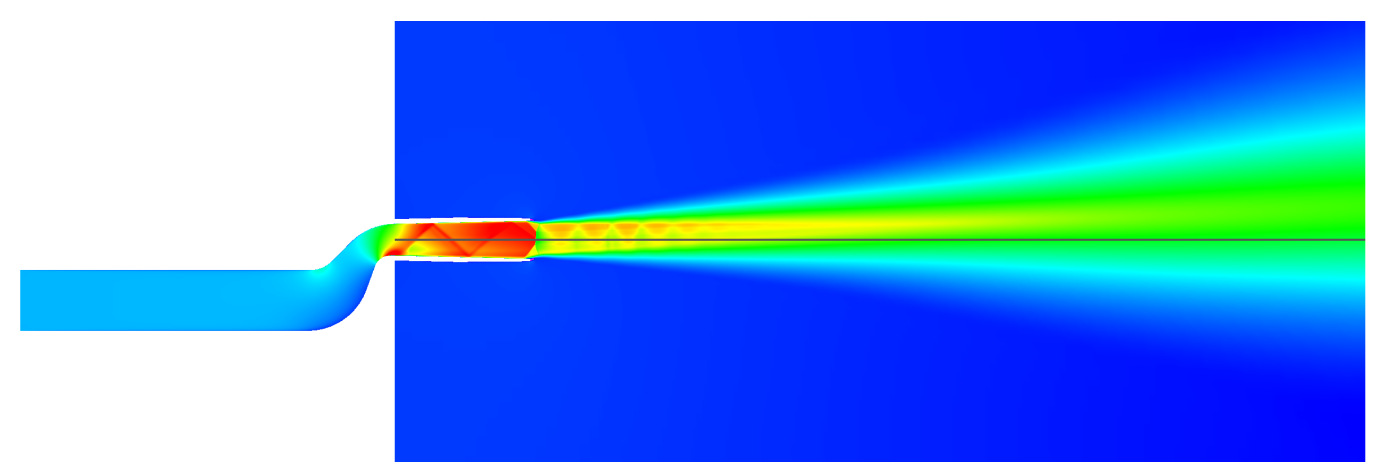

(a) $k$ - $\epsilon$ RANS

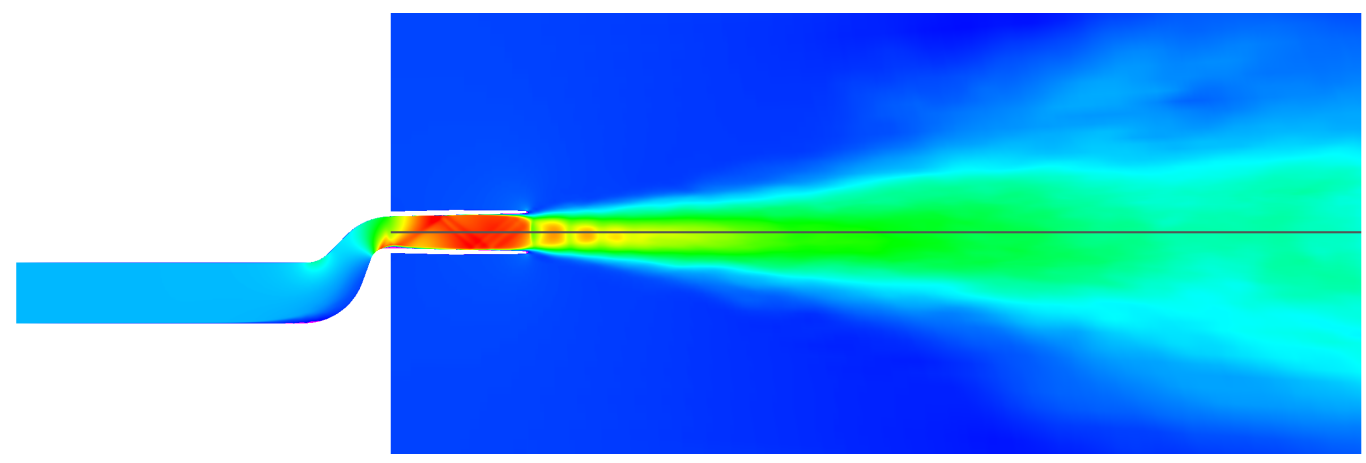

(b) WALE SGS

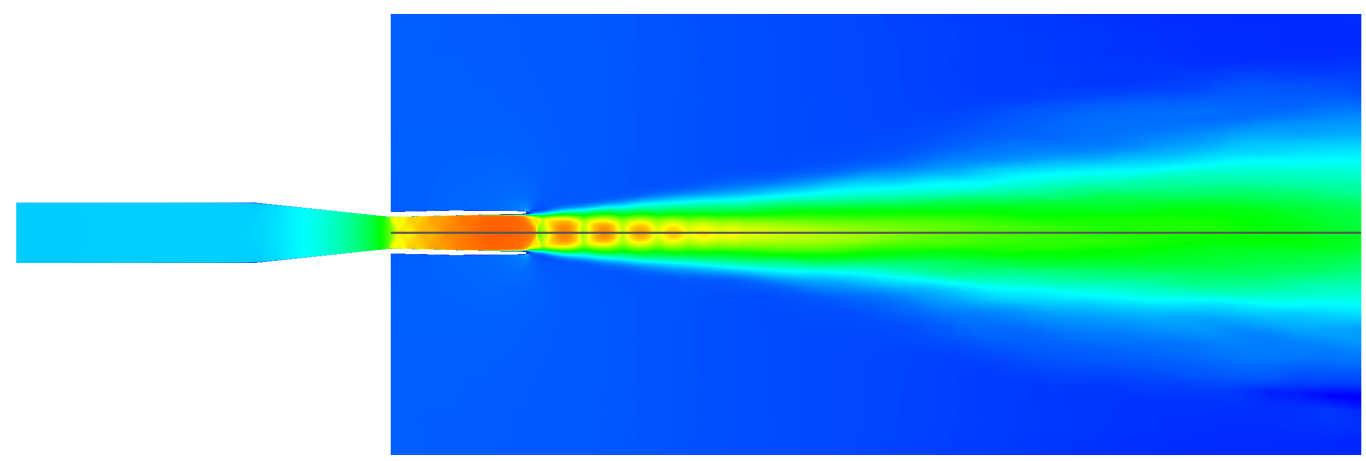

(c) WALE SGS with SEM

$\begin{array}{lllllllllll}-50 & 0 & 50 & 100 & 150 & 200 & 250 & 300 & 350 & 400 & 450\end{array}$

Figure 12: Flow visualisation of axial velocity across the minor axis plane for $70^{\circ} \mathrm{S}$-Duct Curvature. 


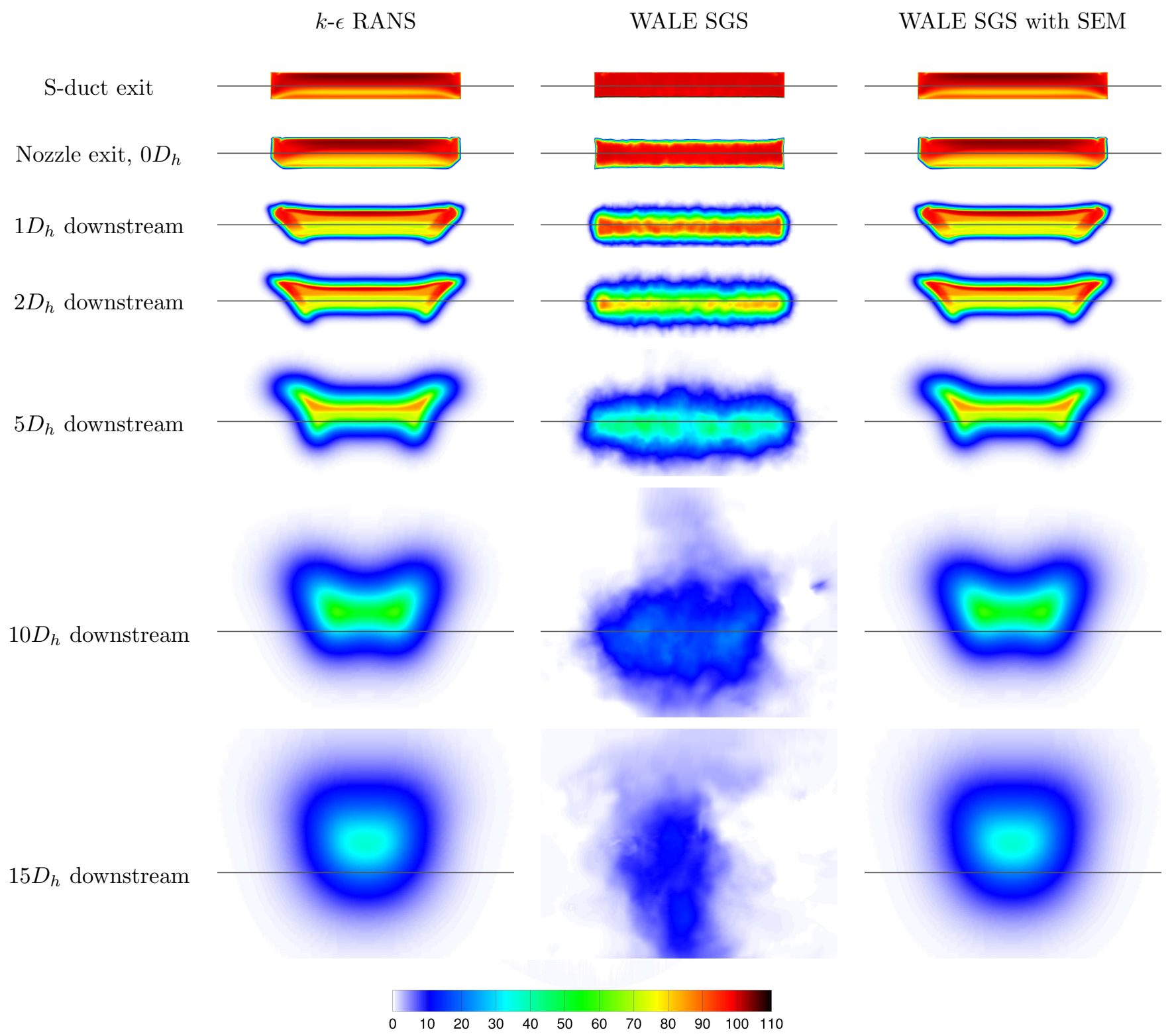

Figure 13: Cross section flow visualisations of percentage total pressure coefficient for $70^{\circ}$ S-Duct Curvature . 


\section{Acknowledgments}

Y. Ng, National University of Singapore, P. Behrouzi and J. McGuirk, Loughborough University, are gratefully acknowledged for their help and for sharing their experimental databases. This work is supported by the EPSRC and Rolls Royce. For computations, this research was supported in part by the Loughborough University Research Computing Service on the Hydra super computer.

\section{References}

${ }^{1}$ Coates, T. D. and Page, G. J., "CFD Based Study of Unconventional Aeroengine Exhaust Systems," AIAA Paper 2012-2775, June 2012.

${ }^{2}$ Miller, D. S., Internal Flow Systems.

${ }^{3}$ Prandtl, L., Prandtl's Essentials of Fluid Mechanics, Springer, 2nd ed., 1953.

${ }^{4}$ Bansod, P. and Bradshaw, P., "The Flow in S-shaped Ducts," Aeronautical Quarterly, Vol. 23, May 1972, pp. 131-140.

${ }^{5} \mathrm{Ng}$, Y. T., Luo, S. C., Lim, T. T., and Ho, Q. W., "On swirl development in a square cross-sectioned S-shaped duct," Experiments in Fluids, Vol. 41, 2006, pp. 975-989.

${ }^{6}$ Taylor, A. M. K. P., Whitelaw, J. H., and Yianneskis, M., "Developing Flow in S-Shaped Ducts I: Square Cross-Section Duct," NASA Contractor Report 3550, May 1982.

${ }^{7}$ Vakili, A., Wu, J. M., Liver, P., and Bhat, M. K., "An Experimental Investigation of Secondary Flows in a S-Shaped Duct," Nasa lewis final report, 1983.

${ }^{8}$ Vakili, A., Wu, J. M., Bhat, M. K., and Liver, P., "Compressible Flow in a Diffusing S-Duct with Flow Separation," Heat Transfer and Fluid Flow in Rotating Machinery, edited by W. J. Yang, Hemisphere Publishing, 1987, pp. $201-211$.

${ }^{9}$ Vakili, A., Wu, J. M., Liver, P., and Bhat, M. K., "Measurements of Compressible Secondary Flow in a Circular S-Duct," AIAA Paper 83-1739, July 1983.

${ }^{10}$ Vakili, A., Wu, J. M., Hingst, W. R., and Towne, C. E., "Comparison of Experimental and Computational Compressible Flow in a S-Duct," AIAA Paper 84-0033, January 1984.

${ }^{11}$ Smith, C. F., Bruns, J. E., Harloff, G. J., and DeBonis, J. R., "Three Dimensional Compressible Turbulent Computations for a Non Diffusing S-Duct," NASA Contractor Report 4391, April 1991.

${ }^{12}$ Smith, C. F., Bruns, J. E., Harloff, G. J., and DeBonis, J. R., "Three Dimensional Compressible Turbulent Computations for a Diffusing S-Duct," NASA Contractor Report 4392, April 1992.

${ }^{13}$ Guo, R. W. and Seddon, J., "The Swirl in an S-Duct of Typical Intake Proportions," Aeronautical Quarterly, Vol. 34, May 1983, pp. 99-129.

${ }^{14}$ Guo, R. W. and Seddon, J., "Swirl Characteristics of an S-Shaped Air Intake with both Horizontal and Vertical Offsets," Aeronautical Quarterly, Vol. 34, May 1983, pp. 130-146.

${ }^{15}$ Klein, M., Sadiki, A., and Janicka, J., "A Digital Filter Based Generation of Inflow Data for Spatially Developing Direct Numerical or Large Eddy Simulations," Journal of Computational Physics, Vol. 186, 2003, pp. 652-665.

${ }^{16}$ Lund, T. S., Wu, X., and Squires, K. D., "Generation of Turbulent Inflow Data for Spatially-Developing Boundary Layer Simulations," Journal of Computational Physics, Vol. 140, 1998, pp. 233-258.

${ }^{17}$ Keating, A., Piomelli, U., Balaras, E., and Kaltenbach, H.-J., "A Priori and a Posteriori Tests Inlet Conditions for Large-Eddy Simulation," Physics of Fluids, Vol. 16, No. 12, December 2004, pp. 4696-4712.

${ }^{18}$ Spalart, P. R., "Direct Simulation of a Turbulent Boundary Layer up to $R e_{\theta}=1410$," Journal of Fluid Mechanics, Vol. 187, 1988, pp. 61-98.

${ }^{19}$ di Mare, L., Klein, M., Jones, W. P., and Janicka, J., "Synthetic Turbulence Inflow Conditions for Large-Eddy Simulation," Physics of Fluids, Vol. 18, No. 02, February 2006, pp. 025107-01-025107-11.

${ }^{20}$ Jarrin, N., Benhamadouche, S., Laurence, D., and Prosser, R., "A Synthetic-Eddy Method for Generating Inflow Conditions for Large-Eddy Simulations," International Journal of Heat and Fluid Flow, Vol. 27, 2006, pp. 585-593.

${ }^{21}$ Jarrin, N., Uribe, J. C., Benhamadouche, S., and Laurence, D., "Reconstruction of Turbulent Fluctuations for Hybrid RANS/LES Simulations Using a Synthetic-Eddy Method," International Journal of Heat and Fluid Flow, Vol. 30, 2009, pp. $435-442$.

${ }^{22}$ Crumpton, P. I., Moinier, P., and Giles, M. G., "An Unstructured Algorithm for High Reynolds Number Flows on Highly Stretched Grids," 10th International Conference on Numerical Methods for Laminar and Turbulent Flow, 1998.

${ }^{23}$ Anderson, B. H., Taylor, A. M. K. P., Whitelaw, J. H., and Yianneskis, M., Developing Flow in S-Shaped Ducts, Vol. 10, Sept-Oct 1982, pp. 661-667. 